\title{
Emulating Earth System Model land temperature fields with MESMER
}

\section{Other Conference Item}

Author(s):

Beusch, Lea (D); Gudmundsson, Lukas (D); Seneviratne, Sonia I. (D)

Publication date:

2021-04

Permanent link:

https://doi.org/10.3929/ethz-b-000528416

Rights / license:

Creative Commons Attribution 4.0 International

Originally published in:

EGUsphere, https://doi.org/10.5194/egusphere-egu21-8640 
EGU21-8640, updated on 26 Jan 2022

https://doi.org/10.5194/egusphere-egu21-8640

EGU General Assembly 2021

(c) Author(s) 2022. This work is distributed under

the Creative Commons Attribution 4.0 License.

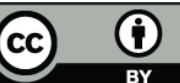

\section{Emulating Earth System Model land temperature fields with MESMER}

Lea Beusch, Lukas Gudmundsson, and Sonia I. Seneviratne

Institute for Atmospheric and Climate Science, ETH, Zurich, Switzerland (lea.beusch@env.ethz.ch)

Earth System Models (ESMs) are invaluable tools to study the climate system's response to greenhouse gas emissions. But their projections are affected by three major sources of uncertainty: (i) internal variability, i.e., natural climate variability, (ii) ESM structural uncertainty, i.e., uncertainty in the response of the climate system to given greenhouse gas concentrations, and (iii) emission scenario uncertainty, i.e., which emission pathway the world chooses. The large computational cost of running full ESMs limits the exploration of this uncertainty phase space since it is only feasible to create a limited number of ESM runs. However, climate change impact and integrated assessment models, which require ESM projections as their input, could profit from a more complete sampling of the climate change uncertainty phase space. In this contribution, we present MESMER (Beusch et al., 2020), a Modular ESM Emulator with spatially Resolved output, which allows for a computationally efficient exploration of the uncertainty space of yearly temperatures. MESMER approximates ESM land temperature fields at a negligible computational cost by expressing grid-point-level temperatures as a function of global mean temperature and an overlaid spatio-temporally correlated variability term. Within MESMER all three major sources of uncertainty can be accounted for. Stochastic simulation of natural climate variability allows to account for internal variability. ESM structural uncertainty can be addressed by calibrating MESMER on different ESMs from the Coupled Model Intercomparison Project (CMIP) archives. Finally, emission scenario uncertainty can be accounted for by ingesting forced global mean temperature trajectories from global climate model emulators, such as MAGICC or FaIR. MESMER is a flexible statistical tool which is under active development and in the process of becoming an open-source software.

Beusch, L., Gudmundsson, L., and Seneviratne, S. I. (ESD, 2020): https://doi.org/10.5194/esd-11-139-2020 